\title{
Inverted hyperplastic polyposis of the colon
}

\author{
N A Shepherd
}

\begin{abstract}
Aims: To describe and evaluate two apparently unique cases of inverted hyperplastic (metaplastic) polyposis of the colon.

Methods: The cases were analysed by standard histopathological, histochemical, and immunohistochemical techniques and the findings compared with those of regular hyperplastic polyps of the colorectum.
\end{abstract}

Results: Both patients were middle-aged men with concurrent adenocarcinoma of the proximal large intestine. The inverted polyps numbered 18 and 12 , measured between 0.4 and $2.5 \mathrm{~cm}$ in diameter, and all were present in the proximal ascending colon. The polyps had characteristic macroscopic features: they were positioned on the apex of mucosal folds and demonstrated surface pitting and mucus hypersecretion. Histologically, inversion and misplacement of hyperplastic epithelium was related to lymphoglandular complexes. The polyps showed all the histochemical and immunohistochemical features of regular hyperplastic polyps. Conclusions: Inverted hyperplastic polyps are an unusual but distinctive polyp of the proximal colon, may be multiple, and share the phenotypic changes of regular hyperplastic polyps. The pathogenesis of epithelial inversion probably relates to misplacement of epithelium through anatomical defects in the muscularis mucosae due to mechanical forces. The polyps may mimic both adenomas and carcinomas. The neoplastic potential of inverted hyperplastic polyposis is likely to be very low: one polyp only showed adenomatous change.

$(\Im$ Clin Pathol 1993;46:56-60)

Hyperplastic (metaplastic) polyps are the commonest polyp of the sigmoid colon and rectum in adults. ${ }^{12}$ Occasionally, multiple polyps are present and in younger patients, particularly men, the term hyperplastic (metaplastic) polyposis has been applied. ${ }^{34}$ Hyperplastic polyps in the left colon and rectum are only very occasionally associated with epithelial misplacement or inversion of the epithelium into the submucosa. ${ }^{3} \mathrm{~A}$ distinctive form of inverted hyperplastic polyp has been described in which epithelial misplacement was a characteristic feature: the polyps were single, predominantly in women, and concentrated in the right colon. ${ }^{5}$

\section{Case reports}

CASE 1

A 68 year old man presented with a three month history of anal bleeding and iron deficiency anaemia. There was no relevant medical or family history. A barium enema and subsequent colonoscopy demonstrated a caecal tumour which was shown on biopsy to be an adenocarcinoma. There were two polyps, each $0.5 \mathrm{~cm}$ in diameter, in the distal colon: both were mildly dysplastic tubular adenomas. Elective right hemicolectomy was performed. The patient was followed up as an outpatient and remained well for nine months after surgery with no signs of tumour recurrence.

\section{CASE 2}

A 62 year old man presented as an emergency with generalised abdominal pain, a high fever, and signs of small intestinal obstruction. There was no relevant medical or family history. At emergency laparotomy a $5 \mathrm{~cm}$ appendiceal mass was discovered with evidence of perforation of the caecum and an acute generalised peritonitis. There was extensive intraperitoneal tumour spread. A palliative extended right hemicolectomy was performed with defunctioning ileostomy and mucus fistula. Despite the extensive tumour, the patient remained well four months after surgery.

\section{Pathology}

All material was received fresh in the laboratory. Resection specimens were opened, pinned out on a corkboard, and fixed in $10 \%$ buffered formalin. Tissues were routinely processed through paraffin wax. Sections were cut at $4 \mu \mathrm{m}$ and stained with haematoxylin and eosin, periodic acid schiff, Perls's stain and high iron diamine alcian blue (HIDAB), the latter according to the method of Spicer. ${ }^{6}$ Sections were stained using the avidin-biotin complex immunohistochemical method with the monoclonal antibodies carcinoembryonic antigen (CEA: Dakopatts), IgA ( $a$ chain; Dakopatts), and secretory component (SC: ICRF, London). Sections with normal colonic mucosa, hyperplastic polyps, and carcinoma served as positive controls. For negative controls primary antibodies were excluded.

\section{CASE 1}

The right hemicolectomy comprised $2 \mathrm{~cm}$ of terminal ileum, caecum with a $9 \mathrm{~cm}$ appendix, and $13 \mathrm{~cm}$ of ascending colon. Arising in the caecum was an ulcerating tumour $6.8 \times 6.3 \mathrm{~cm}$ (fig 1). In the ascending colon within $8 \mathrm{~cm}$ of the ileocaecal valve were a total 
Figure 1 Case 1: the right hemicolectomy specimen contains an ulcerating tumour in the caecum (below), a lipoma in the proximal ascending colon (centre), and multiple inverted

hyperplastic polyps. Even at this magnification the surface puckering of the two largest polyps is evident.

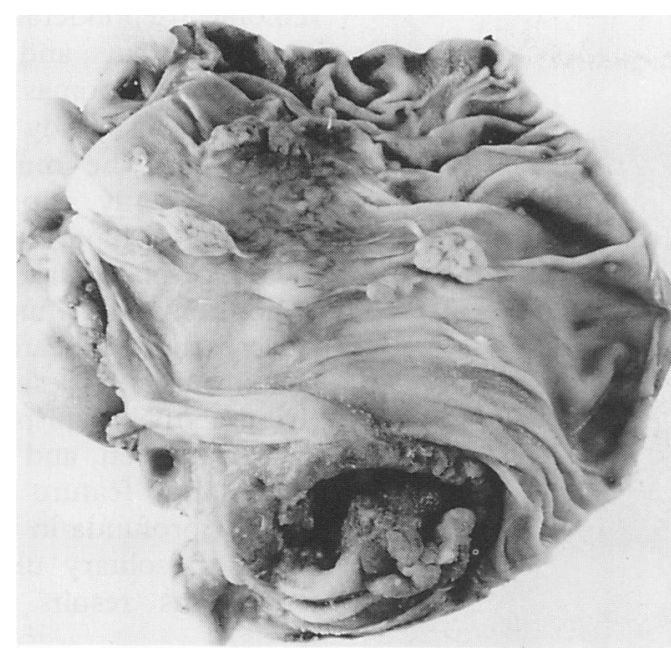

CASE 2

The specimen consisted of $11 \mathrm{~cm}$ of terminal ileum, caecum with a $5 \mathrm{~cm}$ tumour mass replacing the appendix, and $55 \mathrm{~cm}$ of proximal colon. The ileum appeared normal. The ileocaecal valve showed lipohyperplasia. The entire colonic serosa was covered by fibrinous exudate with numerous small tumour nodules. In the proximal ascending colon were a total of 18 polyps. These were transversely orientated on the free edges of mucosal folds. They measured between $0.6 \mathrm{~cm}$ and $2.5 \mathrm{~cm}$ in diameter. They showed very similar macroscopic appearances to the polyps in specimen 1 (fig 2) with a pitted surface and evidence of excessive mucus secretion. The polyps were strictly localised to the proximal $15 \mathrm{~cm}$ of the ascending colon and there were no further mucosal lesions in the more distal colon.

Figure 2 Case 2: three of the polyps from case 2. The polyps are paler than the adjacent mucosa with surface pitting and puckering. They are consistently positioned on the free edge of mucosal folds.

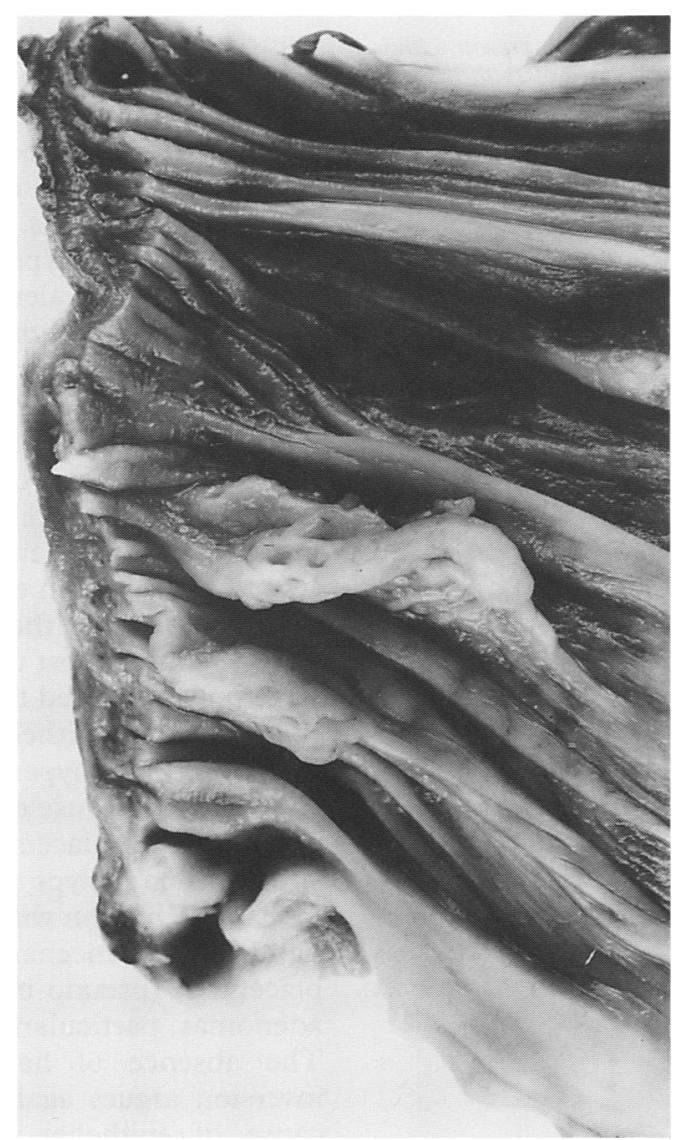

\section{Histological and histochemical findings}

All 30 polyps in both cases showed the architectural, cytological and proliferative features characteristic of hyperplastic polyp. One polyp in case 1 showed a focus of adenomatous epithelium with mild dysplasia adjacent to hyperplastic change, although despite serial sectioning, continuity could not be demonstrated. No other polyp showed such divergent features. In all polyps there were exophytic components characteristic of regular hyperplastic polyp, but in addition all polyps of case 2 and all but three of case 1 showed areas of endophytic growth in which epithelium was embedded in submucosal tissues deep to the muscularis mucosae. Some of these foci took the form of tubules with the characteristic hyperproliferative basal region and serrated maturing epithelium intimately mixed with submucosal connective tissues (figs 3 and 4). Other foci showed the same cellular content but the architecture was grossly distorted by massive mucin hypersecretion and entrapment with the formation of submucosal mucin cysts (fig 5). Mucin hypersecretion was also evident in the mucosal component of these polyps and resulted in a cap of haematoxyphilic mucin on the surface of the polyp (fig 6). Apart from the one focus of tubular adenoma in a polyp of case 1 , dysplastic features were entirely absent. In several foci of endophytic growth, particularly those in which there was relatively early inversion, epithelial misplacement was clearly related to lymphoglandular complexes (fig 3 and 6).

All inverted hyperplastic polyps showed the

of 12 polyps. They were all situated on the surface of mucosal folds, orientated transversely and measured between $0.4 \mathrm{~cm}$ and $2.4 \mathrm{~cm}$ (fig 1 ). The macroscopic appearances of each polyp were similar: they appeared paler than the adjacent mucosa and the surface of the polyp appeared pitted and puckered (fig 1). Several polyps showed evidence of mucus hypersecretion, the surface being covered by viscid mucus. In the ascending colon imme-

- diately beyond the ileocaecal valve was a submucosal lipoma $1.4 \mathrm{~cm}$ in diameter (fig 1). same characteristic mucin histochemical and immunohistochemical profile as that seen in classic hyperplastic polyps. ${ }^{7-9}$ Thus sialomucin was strongly expressed (staining blue with HIDAB), and there was enhanced immunostaining for CEA (fig 7) and diminished staining for SC and IgA in inverted hyperplastic polyps compared with normal colorectal mucosa. Haemosiderin was not demonstrated adjacent to any misplaced epithelium in the Perls preparations. The presence of invasive adenocarcinoma was confirmed in both cases. The caecal tumour of case 1 was a moderately 


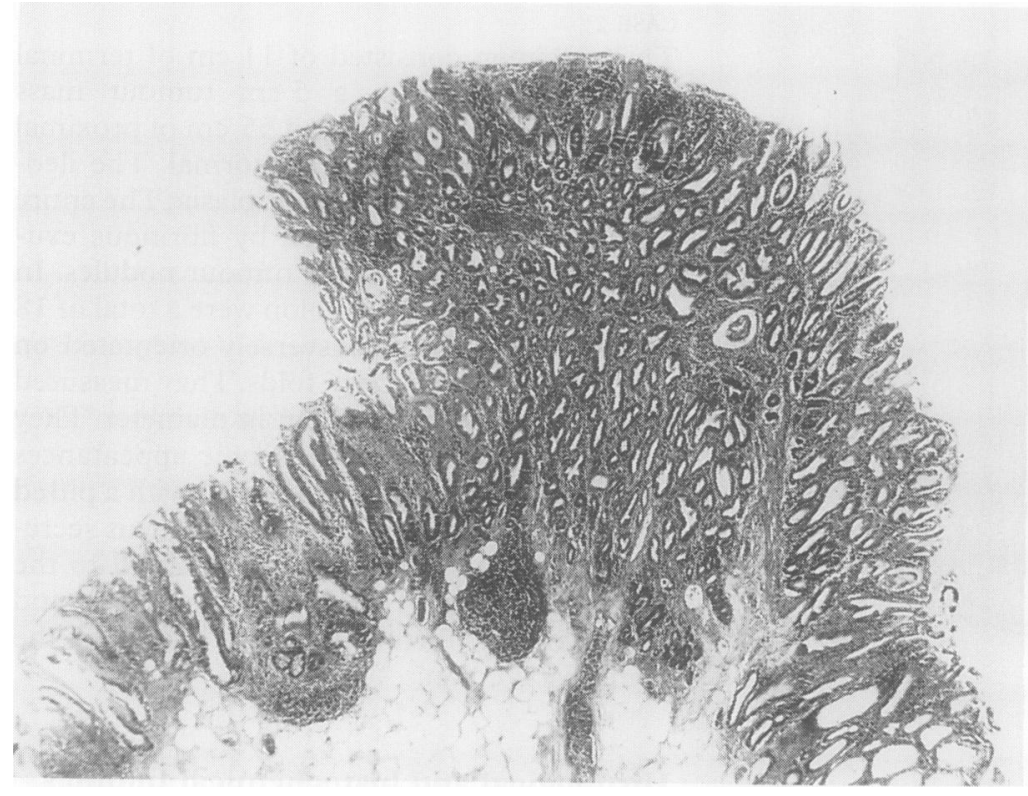

Figure 3 Case 1: an inverted hyperplastic polyp. Proliferative tubules are present within the submucosa. Part of a lymphoid follicle is present adjacent to the area of epithelial misplacement, which is also seen within a lymphoid follicle (at left).

Figure 4 Case 1: the intimate association between hyperplastic epithelial tubules and submucosal fatty tissues.

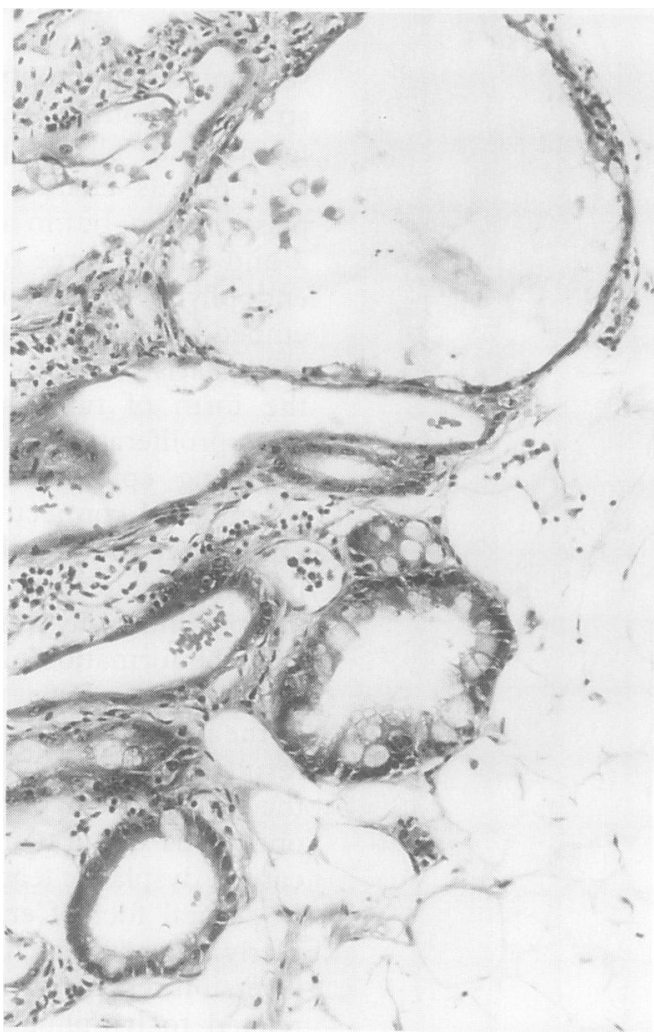

differentiated Dukes B adenocarcinoma with extensive extramural venous spread but no evidence of spread to the peritoneal surface. In case 2 the appendiceal tumour was a poorly differentiated adenocarcinoma, with evidence of serosal pathology and transcoelomic spread.

\section{Discussion}

Sobin's description of solitary inverted hyperplastic polyps emphasises their right-sided colonic preponderance, the predominance of female patients, and their histological mimicry of both adenomas and carcinomas of the colon. ${ }^{5}$ This study clearly shows that such polyps can be multiple and appear to be predominantly situated in the proximal ascending colon: in neither case was there evidence of similar polyps in the more distal colon. This study further shows a characteristic macroscopic appearance with a puckered and pitted surface (presumably the result of underlying epithelial misplacement), surface mucus hypersecretion, and underlying mucous cysts. The latter feature parallels those of colitis cystica profunda in which epithelial misplacement in solitary ulcer syndrome and allied conditions results in submucosal mucous cysts. ${ }^{10}$

In this study the inverted hyperplastic polyps showed morphological, histochemical, and immunohistochemical features identical with those of regular hyperplastic polyps of the sigmoid colon and rectum. ${ }^{7-9}$ They appear to represent the same dysmature and hypermature colonic mucosal lesion ${ }^{39}$ and, like regular hyperplastic polyps, inverted hyperplastic polyps are concentrated on the apices of the mucosal folds. The prime difference between regular hyperplastic polyps and their right-sided equivalents is the frequency of epithelial misplacement and mucus hypersecretion. Another striking feature of these ascending colonic polyps is their relatively large size. Most regular hyperplastic polyps measure under $0.5 \mathrm{~cm},{ }^{3}$ while Sobin records examples of inverted hyperplastic polyps measuring $1.5 \mathrm{~cm}$ in diameter, ${ }^{5}$ and in this study several measured in excess of $2 \mathrm{~cm}$. Could the inversion be solely the result of the size of these polyps? This is most unlikely as both small and large polyps showed the features of inversion in this study and epithelial misplacement is not a feature of giant hyperplastic polyposis. ${ }^{11}$

What is the cause of the epithelial inversion? Epithelial misplacement is seen in several situations in polyps and other lesions of the intestines. Torsion with mucosal necrosis is the pathogenetic mechanism of epithelial misplacement (pseudo-invasion) in pedunculated adenomas, particularly of the sigmoid colon. ${ }^{12}$ The absence of haemosiderin in areas of inversion argues against this mechanism as a cause of epithelial misplacement in hyperplastic polyps. Colitis cystica profunda in solitary ulcer syndrome of the rectum and other conditions in which mucosal prolapse is the pathogenetic mechanism is the result of mucosal erosion and regeneration. ${ }^{10_{1314}}$ Such inflammatory changes are not observed in inverted hyperplastic polyps. In Peutz-Jeghers syndrome up to $10 \%$ of small intestinal polyps show epithelial misplacement. ${ }^{15}$ Obstructive forces with intussusception lead to raised intraluminal pressures and the likely result of this is that the excessive epithelium of the polyps is forced through anatomical defects in the wall of the intestine. ${ }^{15}$ In inverted hyperplastic polyps the epithelial misplacement is seen closely related to lymphoglandular complexes, structures which are associated with an 


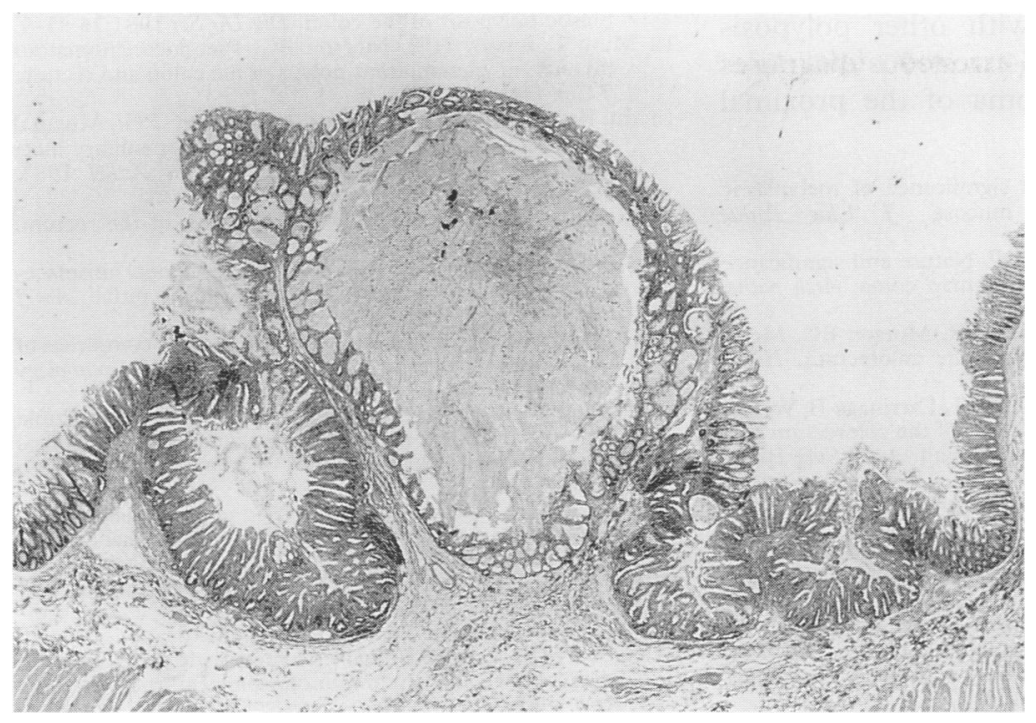

Figure 5 Case 2: a typical large submucosal mucin cyst.

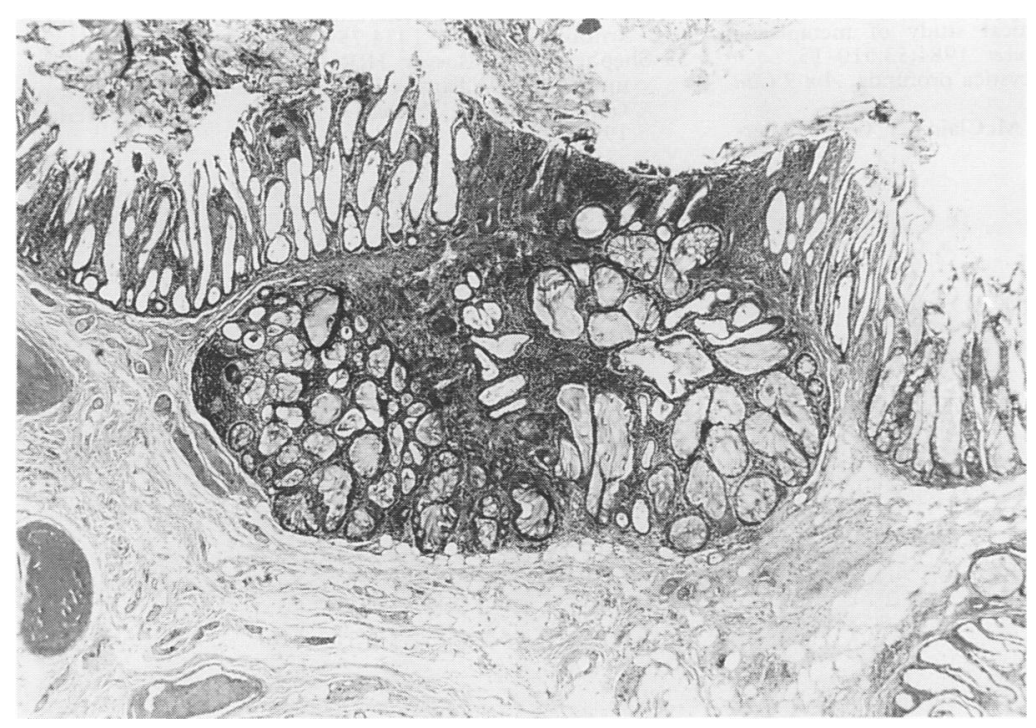

Figure 6 Case 2: epithelial misplacement confined to an enlarged lymphoid follicle within the submucosa. Mucin hypersecretion is well seen both within the dilated misplaced epithelial glands and over the surface of the lesion.

anatomical defect in the muscularis mucosae. ${ }^{16}$ Although lymphoglandular complexes are plentiful in the ascending colon, they are relatively fewer in number here than in the distal colon ${ }^{16}$ and these alone cannot account for the preferential localisation of these lesions to the ascending colon. Nevertheless, the most feasible mechanism for the development of inverted hyperplastic polyps would seem to be mild traction forces, due to the presence of the lesions on the apices of the mucosal folds, leading not to mucosal erosion but to epithelial misplacement through these pre-existing defects in the muscularis mucosae.

Hyperplastic polyposis of the sigmoid colon and rectum is rare and its neoplastic potential is a matter of controversy. ${ }^{3417}$ Evidence against significant carcinogenic potential of hyperplastic polyposis is provided by the recent observation of regression and disappearance of hyperplastic polyposis after surgical removal of rectal cancers, suggesting that the cancers were responsible for, and trophic to, the polyps. ${ }^{18}$

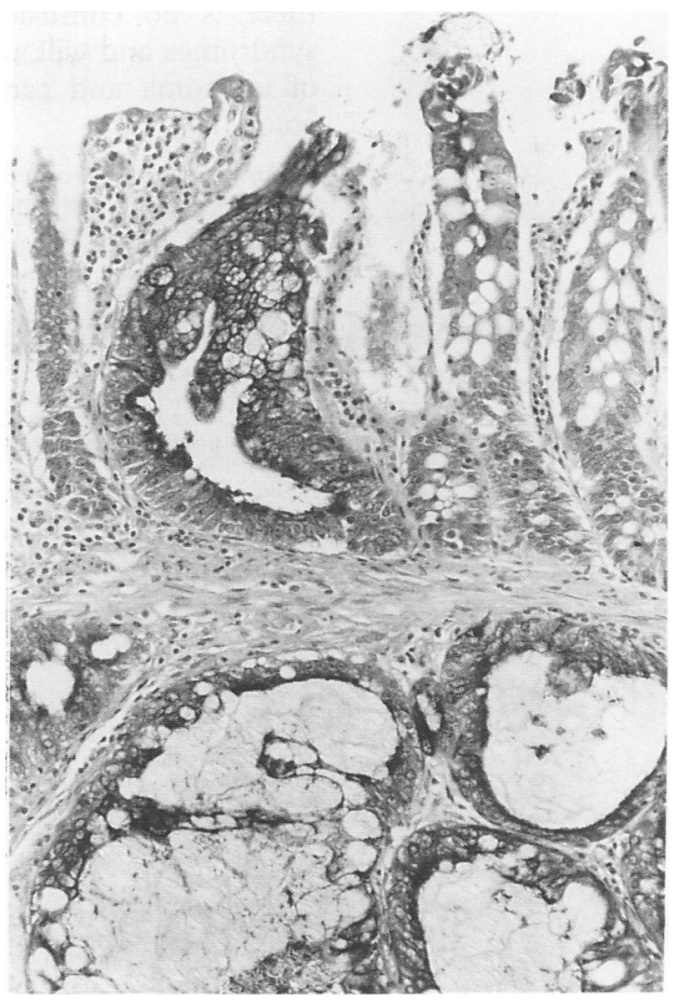

Figure 7 Case 2: enhanced immunostaining for CEA is seen in the hyperplastic surface epithelium (upper left) and the misplaced epithelium (below) compared with the normal colonic mucosa (upper right) (CEA immunohistochemistry).

Just one polyp in the cases under study showed adenomatous change. Such mixed hyperplastic-adenomatous polyps are thought to be associated with malignant potential, ${ }^{19} 20$ but nothing is known of the risk factors for the condition. $^{21}$ There is some evidence to suggest that larger hyperplastic polyps are more likely to show foci of dysplastic/adenomatous epithelium. ${ }^{7}$ Both cases in this study were associated with proximal large intestinal carcinomas but both patients were within the expected age range of such tumours and one at least had a considerably enhanced risk by having two distal colonic adenomas. The inevitable conclusion is that the malignant potential of inverted hyperplastic polyposis is very low and primarily relates to the presence or absence of an associated adenomatous component.

Can the term polyposis be justified for these two cases? In general the term is reserved for syndromes in which large numbers of polyps are present within the bowel. However, some polyposis syndromes, in particular PeutzJeghers and juvenile polyposis, may be associated with relatively low numbers of polyps. Indeed, 10 or more polyps of the juvenile type is an accepted definition for juvenile polyposis. ${ }^{223}$ Inverted hyperplastic polyposis would seem an appropriate term, although it is recognised that these polyps represent a distinctive form of hyperplastic polyp with a characteristic endophytic growth pattern rather than a separate entity. The recognition of the characteristic macroscopic and histological features of these polyps will ensure that 
there is no confusion with other polyposis syndromes and will avoid erroneous diagnoses of adenoma and carcinoma of the proximal colon.

1 Arthur JF. The structure and significance of metaplastic nodules in the rectal mucosa. $f$ Clin Pathol 1968;21:735-43.

2 Goldman H, Ming S, Hickok DF. Nature and significance of hyperplastic polyps of the human colon. Arch pathol 1970;89:349-54.

3 Williams GT, Arthur JF, Bussey HJR, Morson BC. Metaplastic polyps and polyposis of the colorectum. Histopathology 1980;4:155-70.

4 Bengoechea O, Martinez-Penuela JM, Larrinaga B, Valerdi J, Borda F. Hyperplastic polyposis of the colorectum and adenocarcinoma in a 24 year old man. Am $¥$ Surg Pathol 1987;11:323-7.

5 Sobin LH. Inverted hyperplastic polyps of the colon. Am $\mathrm{F}$ Surg Pathol 1985;9:265-72.

6 Spicer SS. The use of various cationic reagents in histochemical differentiation of mucopolysaccharides. $A m$ F Clin Pathol 1961;5:393-407.

7 Franzin G, Zamboni G, Scarpa A, Dina R, Iannucci A Novelli P. Hyperplastic (metaplastic) polyps of the colon. A histological and histochemical study. Am $\mathcal{F}$ Surg Pathol 1984;8:687-98.

8 Jass JR, Faludy J. Immunohistochemical demonstration of IoA and secretory component in relation to epithelial cell differentiation in plastic polyp: plastic polyp: a

9 Jass JR, Filipe MI, Abbas S, Wilson Y, Lovell D. A morphologic and histochemical study of metaplastic polyps of the colorectum. Cancer 1984;53:510-15.

10 Wayte DM, Elwig EB. Colitis cystica profunda. Am $₹$ Clin Pathol 1967;48:159-69.

11 Summer HW, Wasserman NF, McClain CJ. Giant hyper- plastic polyposis of the colon. Dig Dis Sci 1981;26:85-9.

12 Muto T, Bussey HJR, Morson BC. Pseudocarcinomatous invasion in adenomatous polyps of the colon and rectum. invasion in adenomatous poly

13 du Boulay CEH, Fairbrother J, Isaacson PG. Mucosal prolapse syndrome - a unifying concept for solitary ulcer syndrome and allied conditions. F Clin Pathol 1983 36:1264-8.

14 Madigan MR, Morson BC. Solitary ulcer of the rectum Gut 1969;19:871-8.

15 Shepherd NA, Bussey HJR, Jass JR. Epithelial misplacement in Peutz-Jeghers polyps: a diagnostic pitfall. $A m^{\prime}$ Surg Pathol 1987;11:743-9.

16 O'Leary AD, Sweeney EC. Lympho-glandular complexes of the colon: structure and distribution. Histopathology 1986;10:267-84.

17 Cappell MS, Forde KA. Spatial clustering of multiple hyperplastic, adenomatous and malignant colonic polyps
in individual patients. Dis Colon Rectum 1989; in individua

18 Kusunoki M, Fujita S, Sakanoue Y, et al. Disappearance of hyperplastic polyposis after resection of rectal cancer. $D i$ Colon Rectum 1991;34:829-31.

19 Cooper HS, Patchefsky AS, Marks G. Adenomatous and carcinomatous changes within hyperplastic colonic epithelium. Dis Colon Rectum 1979;22:152-6.

20 Gebbers J-O, Laissue JA. Mixed hyperplastic and neoplastic polyp of the colon. An immunohistological study. Virchows Arch 1986;410:189-94.

21 Fenoglio-Preiser CM, Lantz PE, Listrom MB, Davis $M$ Rilke FO. Polyposis In:Gastrointestinal pathology. An atlas and text. New York: Raven Press, 1989:503.

22 Sachatello CR, Hahn IS, Carrington CB. Juvenile gastrointestinal polyposis in a female infant. Report of a case and review of the literature of a recently recognised and review of the literature of a

23 Shepherd NA, Bussey HJR. Polyposis syndromes - an update. In: Williams GT, ed. Current topics in pathology Gastrointestinal Pathology. Berlin: Springer-Verlag, 1990:323-52. 